\title{
Precise Tip Positioning of a Flexible Manipulator using Resonant Control
}

\author{
I. A. Mahmood, S. O. R. Moheimani and B. Bhikkaji
}

\begin{abstract}
A single-link flexible manipulator is fabricated to represent a typical flexible robotic arm. This flexible manipulator is modeled as a SIMO system with the motor-torque as the input and the hub angle and the tip position as the outputs. The two transfer functions are identified using a frequencydomain system identification method. A feedback loop around the hub angle response with a Resonant controller is designed to damp the resonant modes. A high gain integral controller is also designed to achieve zero steady-state error in the tip position response. Experiments are performed to demonstrate the effectiveness of the proposed control scheme.
\end{abstract}

\section{INTRODUCTION}

Increasing demands for high speed manipulation and high payload to weight ratio in robot manipulators has triggered a significant growth in research and development activities on flexible manipulators. These manipulators constitute a suitable choice to realize such demands since they are light in weight, require small sized actuators and consume low energy for actuation [1]. However, designing feedback controllers to operate these systems at high speeds is a challenging task. The control system must be designed not only for precise tip positioning but also for suppressing vibrations associated with the flexible nature of the manipulator.

A wide range of control schemes such as linear quadratic gaussian (LQG) [2], linear quadratic regulator (LQR) [3], $H_{\infty}$ control [4] and $\mu$-synthesis [5] have been used for the positioning of flexible manipulators. In [2], Cannon and Schmitz designed an LQG controller and used measurements from a noncollocated tip position sensor as the controller input. Their results suggested a satisfactory step response with accurate tip positioning. However, the LQG controller was not robust with respect to modeling errors and was of a very high order. In [6], the authors improved the closed-loop system robustness of [2] by wrapping a second feedback loop, consisting of an $H_{\infty}$ controller around the controlled system. The $H_{\infty}$ controller was designed for the purpose of incorporating robustness and also for attenuating disturbances. Simulation results suggested that the control scheme was more robust to uncertainties such as modeling errors. The lack of robustness demonstrated in [2], and [6] is believed to be due to the use of noncollocated sensors which result in non-minimum phase systems [7]. Nevertheless, the noncollocated sensors are often used as they are needed for precise tip positioning.

The authors are with the School of Electrical Engineering and Computer Science, The University of Newcastle, Callaghan, NSW 2308, Australia. R. Moheimani is the corresponding author. Reza.Moheimani@newcastle.edu.au
In contrast to the research reported in the above references, vibration control was given precedence over tip positioning in [8] and [9]. In [8] the author proposed a direct strain feedback (DSFB) control strategy to introduce a damping term into the differential equation governing the vibration of the flexible manipulator. This control strategy managed to increase the stiffness of the flexible manipulator and caused it to undergo smaller vibration levels while in motion. In [9], a sliding mode controller was formulated to control the tip position of a flexible manipulator subjected to parameter variations. The authors showed via simulations that the controller performed better in regulating vibrations when initial conditions were incorporated into the designed controller.

In this work, an experimental flexible manipulator setup is fabricated to represent a typical flexible robotic arm. A control scheme is developed such that the vibration suppression is achieved using a collocated measurement and tip positioning is done using a noncollocated measurement. The control scheme consists of two feedback loops with each feedback loop having a specific purpose. The inner loop contains a Resonant controller to add damping to the flexible manipulator. The Resonant controller utilizes the hub angle measurement provided by a shaft encoder and guarantees that the closed-loop system remains stable in the presence of out-of-bandwidth dynamics, [10] and [11]. In the outer loop, using tip position measurements, an integral controller is implemented for precise tip positioning. The integral controller ensures zero steady-state error for a step input.

Successful utilizations of Resonant controllers for vibration suppression in flexible structures have been reported in [10], [11] and [12]. This paper reports the first-time application of this control design approach to flexible manipulators. At the time of this writing it is not known how an optimal Resonant controller can be designed. This is mainly due to the non-convex nature of the optimization problem associated with minimization of a specific performance index. In this paper a graphical approach is proposed, which results in Resonant controllers with satisfactory performance.

The remainder of the paper is arranged as follows. Section II provides a description of the experimental setup. System identification of the system transfer-functions are presented in Section III. Control schemes are devised in Section IV. In Section V, simulation and experimental results are presented to illustrate the effectiveness of the proposed control schemes. Finally, the paper is concluded in Section VI. 




Fig. 1. Flexible manipulator

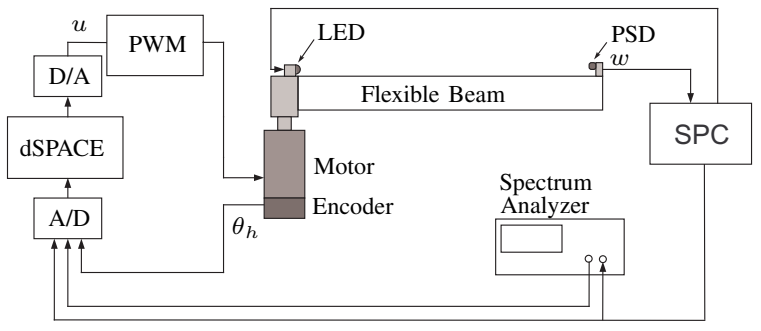

Fig. 2. Experimental setup for the flexible manipulator

TABLE I

MECHANICAL PROPERTIES OF THE FLEXIBLE MANIPULATOR

\begin{tabular}{|c|c|}
\hline Properties & Values \\
\hline Length, $L$ & $0.6 \mathrm{~m}$ \\
\hline Thickness, $h \mathrm{q}$ & $0.003 \mathrm{~m}$ \\
\hline Width, $v$ & $0.05 \mathrm{~m}$ \\
\hline Linear density, $\rho$ & $0.3975 \mathrm{~kg} / \mathrm{m}$ \\
\hline Radius of hub, $r$ & $0.025 \mathrm{~m}$ \\
\hline Modulus of elasticity, $E$ & $6.894 \times 10^{10} \mathrm{~Pa}$ \\
\hline $\begin{array}{c}\text { Hub moment of inertia } \\
\text { (including motor), } I_{h}\end{array}$ & $1.850 \times 10^{-3} \mathrm{Kg} \cdot \mathrm{m}^{2}$ \\
\hline
\end{tabular}

\section{EXPERIMENTAL SETUP}

The experiments were performed in the Laboratory for Dynamics and Control of NanoSystems at The University of Newcastle, Australia. The flexible manipulator used here consists of an aluminum beam clamped directly to the shaft of a Glentek GM4040-41 DC brush servo motor. A detailed illustration of the experimental setup is presented in Fig. 1 and 2. The dimensions and the mechanical properties of the beam are given in Table I. The motor was driven by a Glentek GA377 pulse width modulation (PWM) servomotor amplifier. The shaft encoder of the motor was used to measure the hub angle of rotation. The shaft encoder has a count of 5000 per revolution, i.e. a resolution of 0.072 degrees.

An infrared light-emitting diode (LED) and a Hamamatsu S1352 position sensitive detector (PSD) were used for measuring the deflection of the tip of the beam. A dSPACE DS1103 controller board was used for real-time controller implementation. A sampling frequency of $20 \mathrm{kHz}$ was used in order to avoid aliasing.

\section{SYSTEM IDENTIFICATION}

In this work, an experimental approach is taken to model the dynamics of the flexible manipulator. The following

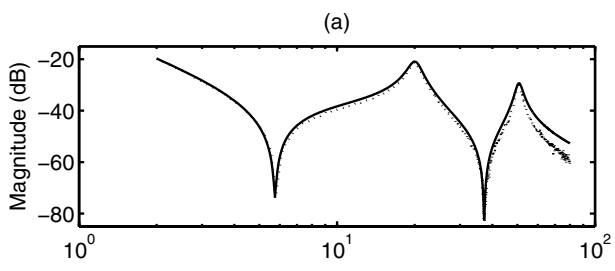

(b)

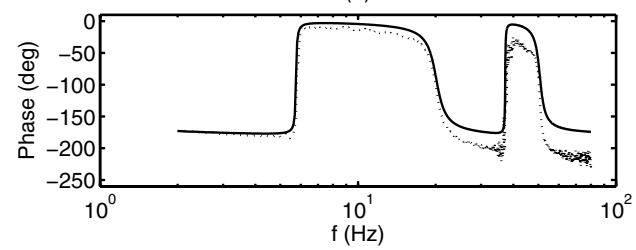

Fig. 3. Identified model (-) and experimental $(\cdots)$ frequency response of amplifier input voltage $u$ to hub angle $\theta_{h}$.

(a)

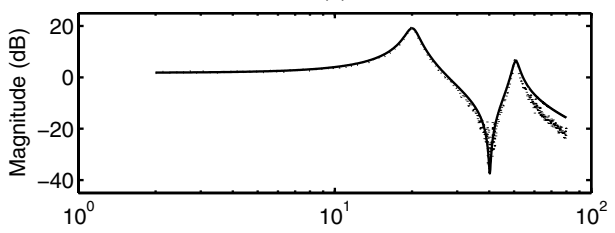

(b)

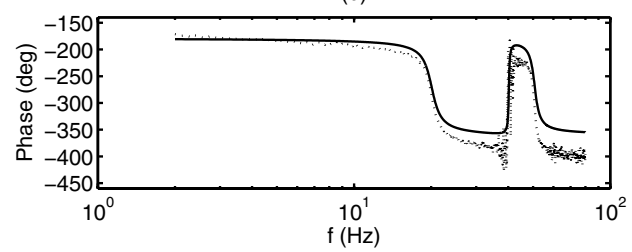

Fig. 4. Identified model $(-)$ and experimental $(\cdots)$ frequency response of amplifier input voltage $u$ to tip deflection $w_{t i p}$.

frequency response functions (FRFs) are determined for designing the control system:

$$
\begin{aligned}
G_{\theta_{h} u}(\mathrm{i} \omega) & \triangleq \frac{\theta_{h}(\mathrm{i} \omega)}{u(\mathrm{i} \omega)}, \\
G_{w_{t i p} u}(\mathrm{i} \omega) & \triangleq \frac{w_{t i p}(\mathrm{i} \omega)}{u(\mathrm{i} \omega)}
\end{aligned}
$$

and

$$
G_{y_{t i p} u}(\mathrm{i} \omega)=G_{w_{t i p} u}(\mathrm{i} \omega)+L G_{\theta_{h} u}(\mathrm{i} \omega)
$$

where $u(t)$ is the input voltage, $\theta_{h}(t)$ is the hub angle measured by the shaft encoder, $w_{t i p}(t)=w(L, t)$ is the flexural tip deflection measured by the PSD. It is worth noting that the tip position $y_{t i p}(t) \triangleq y(L, t)=w(L, t)+L \theta_{h}(t)$, which leads to the expression (3) for the FRF $G_{y_{t i p} u}(\mathrm{i} \omega)$.

A dual channel HP35670A spectrum analyzer was used for determining the FRFs. A band limited random noise signal ( 2 to $102 \mathrm{~Hz}$ ) was generated using the spectrum analyzer and applied to the motor as the input, $u(t)$. The corresponding outputs $\theta_{h}(t)$ and $w_{t i p}(t)$ were also recorded using the spectrum analyzer. The input-output data was processed to generate the FRFs (1) and (2) in a non-parametric form. In Fig. 3 and 4 the nonparametric FRFs of (1) and (2) are plotted 
along with corresponding parametric fits,

$$
\begin{aligned}
G_{\theta_{h} u}(s)= & \frac{420.73\left(s^{2}+0.5028 s+1305\right)}{s(s+1.65)\left(s^{2}+15.35 s+1.596 \times 10^{4}\right)} \\
& \times \frac{\left(s^{2}+1.437 s+5.462 \times 10^{4}\right)}{\left(s^{2}+20.9 s+1.015 \times 10^{5}\right)}
\end{aligned}
$$

and

$$
\begin{aligned}
G_{w_{t i p} u}(s)= & \frac{-31153.01}{\left(s^{2}+15.35 s+1.596 \times 10^{4}\right)} \\
& \times \frac{\left(s^{2}+3.108 s+6.386 \times 10^{4}\right)}{\left(s^{2}+20.9 s+1.015 \times 10^{5}\right)} .
\end{aligned}
$$

Note that the poles characterizing the flexible modes of the beam in $G_{\theta_{h} u}(s)$ and $G_{w_{t i p} u}(s)$ are identical. Data beyond $80 \mathrm{~Hz}$ were discarded as these frequencies were far beyond the maximum bandwidth of the motor (which is close to $60 \mathrm{~Hz}$ ). Fig. 3, clearly illustrates the collocated nature of $G_{\theta_{h} u}(s)$.

\section{CONTROLLER DESIGN}

This section discusses and details the control design scheme proposed in this paper. The control scheme consists of two negative feedback loops.

\section{A. Resonant controller design (Inner loop controller)}

Feedback controllers which increase the effective damping and at the same time guarantee unconditional stability of the closed-loop system are always preferred, as they avoid closed-loop instabilities due to spill-over effects [13]. It is known that a collocated velocity feedback controller [13] possess such properties. However, the implementation of this controller requires the realization of a differentiator, which is not possible for systems with large bandwidth. Another drawback of the velocity feedback controller is that it results in a high control effort at all frequencies. Ideally, for vibration damping purposes, the control effort should be restricted to the resonance frequencies only. Resonant controllers are a class of controllers that guarantee unconditional closedloop stability of collocated systems. The model structure of Resonant controllers are such that they approximate a differentiator over a narrow bandwidth around the resonance frequencies. Resonant controllers were first proposed in [10]. The motivations for their model structure comes from passive RL network controllers for piezoelectric shunt damping, see [14], [15] and [16].

As the poles characterizing the flexible modes of $G_{\theta_{h} u}(s)$ and $G_{y_{t i p} u}(s)$ are identical, system resonances can be damped by designing a feedback loop around either $G_{\theta_{h} u}(s)$ or $G_{y_{t i p} u}(s)$. Here, $G_{\theta_{h} u}(s)$ is chosen due to its collocated nature. Damping can be achieved by shifting the closed-loop poles of $G_{\theta_{h} u}(s)$ deeper into the left half plane.

In general, a resonant controller is defined as

$$
K(s)=\sum_{i=1}^{N} \frac{\alpha_{i} s^{2}}{s^{2}+2 \delta_{i} \omega_{i} s+\omega_{i}^{2}}
$$

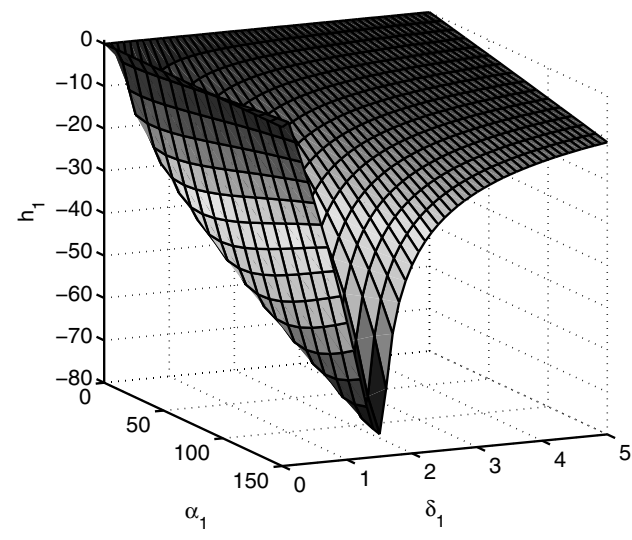

Fig. 5. Plot of distance between the real parts of the open-loop and closeloop poles $h_{1}$ versus $\alpha_{1}$ and $\delta_{1}$, for the 1st flexible mode.

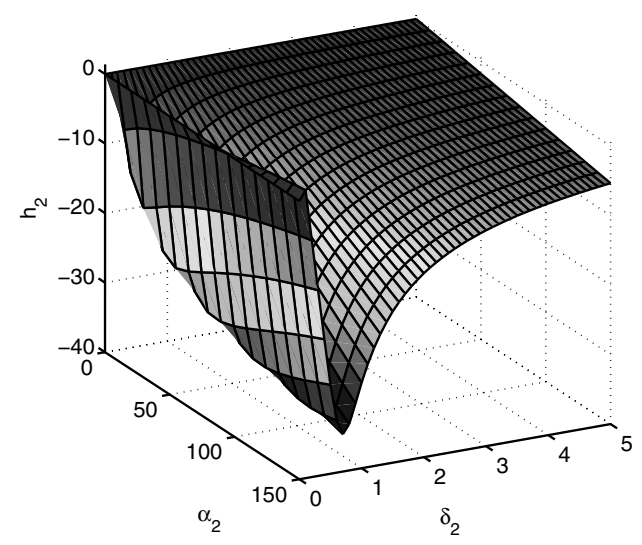

Fig. 6. Plot of distance between the real parts of the open-loop and closeloop poles $h_{2}$ versus $\alpha_{2}$ and $\delta_{2}$, for the 2nd flexible mode.

where $\alpha_{i}, \delta_{i}$ and $\omega_{i}$ are the design parameters, and $N$ is the number of modes that need to be controlled. In the current context $N=2$, which implies

$$
K(s)=K_{1}(s)+K_{2}(s),
$$

where

$$
K_{1}(s)=\frac{\alpha_{1} s^{2}}{s^{2}+2 \delta_{1} \omega_{1} s+\omega_{1}^{2}}
$$

and

$$
K_{2}(s)=\frac{\alpha_{2} s^{2}}{s^{2}+2 \delta_{2} \omega_{2} s+\omega_{2}^{2}} .
$$

Here, the resonant filters $K_{1}(s)$ and $K_{2}(s)$ are determined independently. Each $\omega_{i}$ is set to the $i^{\text {th }}$ natural frequency of the flexible manipulator and the value of $\alpha_{i}$ and $\delta_{i}$ are varied such that the absolute distance between the real parts of the open-loop and closed-loop poles, $h_{i}$, is maximized. Fig. 5 and 6 show that for a given range of $\alpha_{i}\left(0 \leq \alpha_{i} \leq 150\right)$, there exists a value of $\delta_{i}$ which maximizes the absolute value of $h_{i}$. The controller obtained for this range of $\alpha$ is

$$
\begin{aligned}
K(s)= & \frac{150 s^{2}}{s^{2}+378.3 s+1.59 \times 10^{4}} \\
& +\frac{150 s^{2}}{s^{2}+445.8 s+1.014 \times 10^{5}} .
\end{aligned}
$$


Better damping can be achieved by increasing the range of $\alpha$. However, this would result in a controller that has a higher gain. The high magnitude could amplify highfrequency noise which could lead to degradation of the closed-loop performance. Here, it is possible to determine the resonant filters independently because there is sufficient frequency spacing between the system poles and zeros and the action of the resonant filters is mostly uncoupled [10].

It is worth noting that in closed-loop, the Resonant controller $K(s)$ will not shift the pole located at the origin. This can be seen by setting

$$
G_{\theta_{h} u}(s)=\frac{a(s)}{s \times b(s)}
$$

and

$$
K(s)=\frac{s^{2} \times p(s)}{q(s)}
$$

and noting that

$$
\begin{aligned}
G_{\theta_{h} u}^{(c l)}(s) & =\frac{G_{\theta_{h} u}(s)}{1+K(s) G(s)} \\
& =\frac{1}{s}\left(\frac{a(s)}{q(s) b(s)+s p(s) a(s)}\right) .
\end{aligned}
$$

\section{B. Outer loop for positioning}

Integral controller was implemented in the outer feedback loop to provide precise tip positioning. In designing the integral controllers, the gross response of the flexible manipulator to a step input needs to satisfy the following specifications: 1) Zero steady-state tip position error, 2) Rise time and settling time of less than 1 and $1.5 \mathrm{~s}$, respectively and 3) Overshoot of less than $2 \%$.

An important property of an integral controller is that a positive error will always result in increasing the control signal while a negative error will result in a decreasing control signal, regardless of the magnitude of the error, [17]. This property is desirable because the error introduced by the motor friction, which becomes visible when the tip is nearing the given set-point or moving at a very slow speed, can be eliminated.

In order to wrap an integral controller around $G_{y_{t i p} u}(s)$ such that the resulting closed-loop has an acceptable stability margin, $G_{\theta_{h} u}^{(c l)}(s)$ must not have a pole at the origin. This can be checked using the standard root-locus criterion. A simple way to correct this problem would be to add or augment the Resonant controller with a rational function $C(s)$. In other words the pure Resonant controller in the inner feedback loop is replaced by

$$
K_{a}(s)=K(s)+C(s),
$$

see also the illustration in Fig. 7. In order to avoid a large increase in the model order of the controller and at the same time push the pole at $s=0$ well into the left half plane, a phase-lead compensator is used,

$$
C(s)=\frac{K_{p l}(s+\alpha)}{s+\beta}
$$

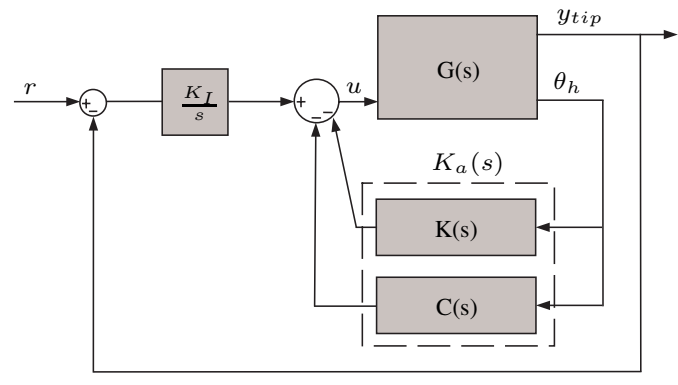

Fig. 7. Augmented Resonant controller $K_{a}(s)$ and integral controller $\frac{K_{I}}{s}$.

where $K_{p l}, \alpha$ and $\beta$ are the design parameters. The parameters can be chosen using root-locus approach. The use of of phase-lead compensator and guidelines on pole placement using them are given in detail in [18].

Here we set

$$
C(s)=\frac{70(s+10)}{s+70}
$$

which implies that the augmented Resonant controller is equal to

$$
\begin{aligned}
K_{a}(s)= & \frac{70(s+10)}{s+70}+\frac{150 s^{2}}{s^{2}+378.3 s+1.59 \times 10^{4}} \\
& +\frac{150 s^{2}}{s^{2}+445.8 s+1.014 \times 10^{4}} .
\end{aligned}
$$

\section{SIMULATION AND EXPERIMENTAL RESULTS}

This section presents simulation and experimental results obtained from the control schemes proposed in this paper.

\section{A. Resonant and Integral controller}

The effect of damping introduced by the augmented Resonant controller $K_{a}(s)$ on the resonant modes were first evaluated. In Fig. 8 the simulated closed-loop frequency response of $G_{\theta_{h} u}(s)$ is plotted along with its experimental counterpart. It is evident that the experimental results match the simulations except near the second resonant mode. This is due to the fact that the second resonance is very close to the maximum bandwidth of the motor.

In Fig. 9, experimentally determined closed-loop frequency responses of $G_{\theta_{h} u}(s)$ and $G_{w_{t i p} u}(s)$ are plotted along with their corresponding open-loop frequency responses. A significant damping in the first and the second resonances of both $G_{\theta_{h} u}(s)$ and $G_{w_{t i p} u}(s)$ is evident from the plots. In particular, Fig. 9 (a) illustrates $20 \mathrm{~dB}$ and $19 \mathrm{~dB}$ damping on the 1st and 2nd modes of $G_{\theta_{h} u}(s)$ respectively. Furthermore, Fig. 9 (b) shows damping of $18 \mathrm{~dB}$ on the $1^{\text {st }}$ and $2^{\text {nd }}$ mode of $G_{w_{t i p} u}(s)$, respectively.

Having the flexible manipulator significantly damped by the augmented Resonant controller, experiments were performed to slew the tip to a set-point $y_{t i p}=\frac{\pi L}{4} \mathrm{~m}$, with the present position being set to zero. For the sake of comparison, initially, the tip was slewed in open-loop to obtain the open-loop time response of the tip position and tip deflection. The amount of time the input voltage $u$ needed 


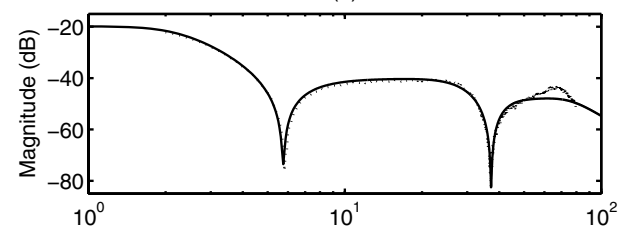

(b)

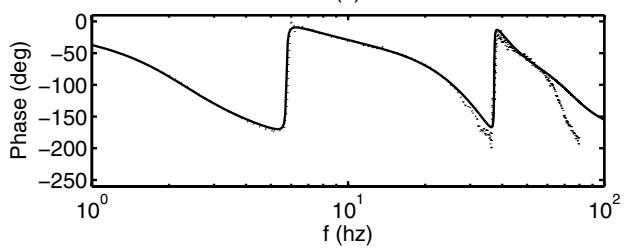

Fig. 8. Simulated (-) and experimental $(\cdots)$ closed-loop frequency responses of amplifier input voltage $u$ to hub angle $\theta_{h}$ using augmented Resonant controller $K_{a}(s)$.

(a)

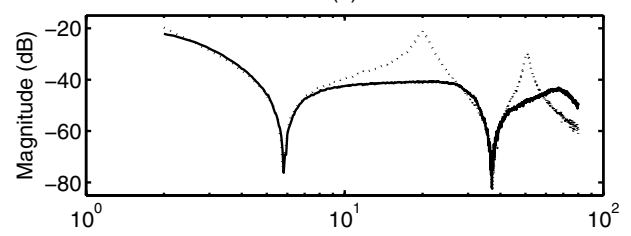

(b)

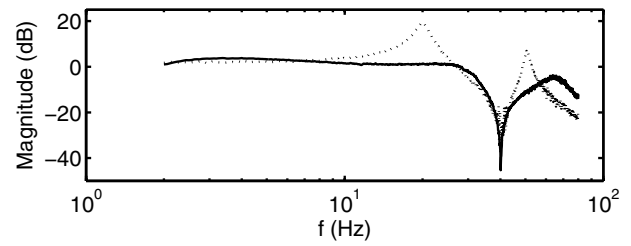

Fig. 9. Open-loop $(.$.$) and closed-loop (-): Frequency responses of$ (a) amplifier input voltage $u$ to hub angle $\theta_{h}$, (b) amplifier input voltage $u$ to tip deflection $w_{t i p}$ using augmented Resonant controller $K_{a}(s)$.

to be applied to the motor in order to slew the tip to the setpoint was determined through simulation. Fig. 10 illustrates that the open-loop control resulted in a tip position response with a large steady-state error, slow rise and settling times, and a highly oscillating tip.

Similar slewing experiments were performed with an integral controller in the outer feedback loop. Here, rootlocus approach was used in selecting the integral controller gain $K_{I}$. Fig. 11 (a) shows the closed-loop time response of tip position $y_{\text {tip }}$ with $K_{I}=30$. It is apparent from the plot, that $y_{\text {tip }}$ has a zero steady-state error, a zero overshoot, a rise time of $0.5 \mathrm{~s}$ and a settling time of $1.0 \mathrm{~s}$. The high gain in $K_{I}$ has allowed the tip position to have zero steady-state error in $1.3 \mathrm{~s}$. Fig. 11 (b) illustrates that the Resonant controller completely suppresses the tip vibrations during, and at the end of the slewing maneuver.

A faster response of $y_{t i p}$ can be obtained by increasing the value of $K_{I}$, but this comes at the expense of a higher overshoot. Fig. 12 (a) shows the response $y_{\text {tip }}$ when $K_{I}$ is increased to 45 . It can be observed that the rise and settling times have decreased to $0.2 \mathrm{~s}$ and $0.6 \mathrm{~s}$ respectively, while the overshoot has increased from 0 to $6.6 \%$. It is worth noting
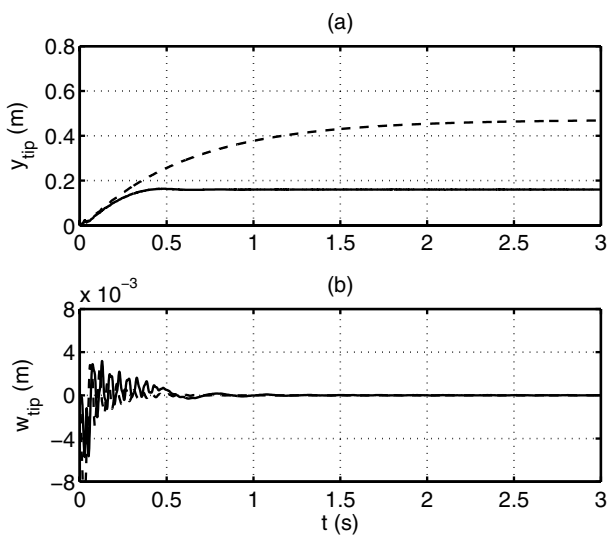

Fig. 10. Experimental (-) and simulated (--): Time response plots of (a) Tip position $y_{t i p}$, (b) Tip deflection $w_{t i p}$, in open-loop.

(a)
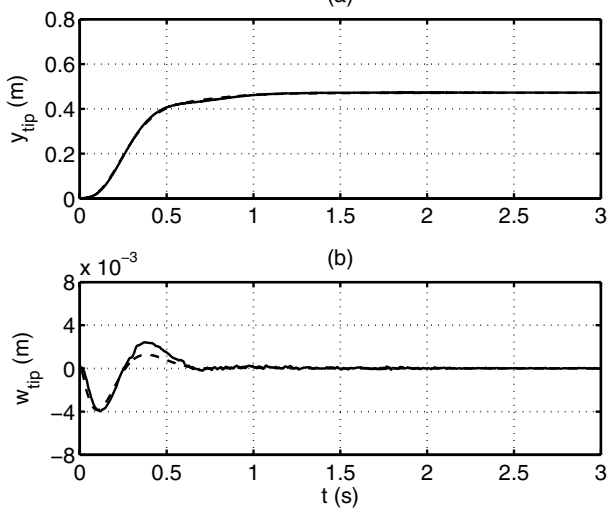

Fig. 11. Experimental (-) and simulation (--): Time response plots of (a) Tip position $y_{t i p}$, (b) Tip deflection $w_{t i p}$, using augmented Resonant controller $K_{a}(s)$ and integral controller for $K_{I}=30$.
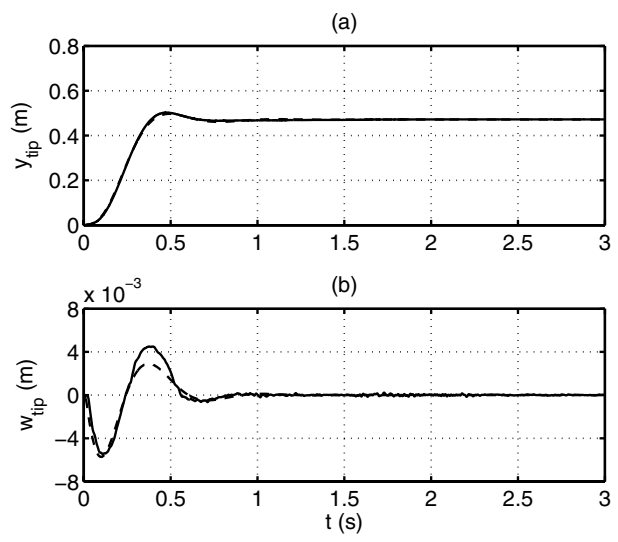

Fig. 12. Experimental (-) and simulation (--): Time response plots of (a) Tip position $y_{t i p}$, (b) Tip deflection $w_{t i p}$, using augmented Resonant controller $K_{a}(s)$ and integral controller for $K_{I}=45$.

that, even for a faster tip position response, Fig. 12 (b) does not show any indication of tip vibrations.

\section{B. Robustness analysis}

The robustness of the proposed controller scheme is analyzed here. The first robustness test was performed by 
(a)
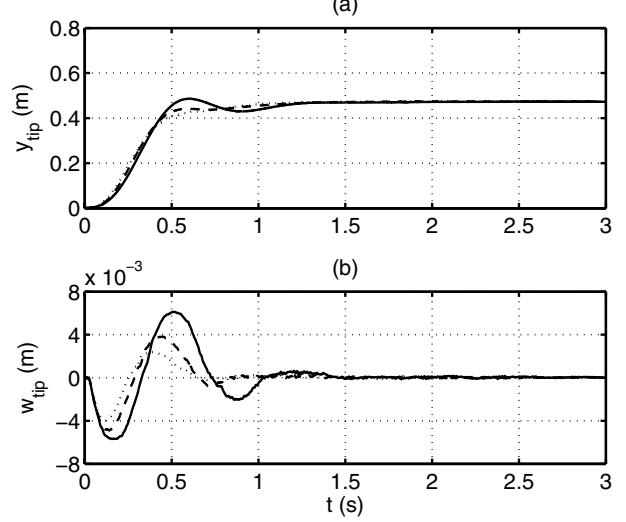

Fig. 13. Time response plots of (a) Tip position $y_{t i p}$, (b) Tip deflection $w_{\text {tip }}$, using augmented Resonant controller $K_{a}(s)$ and integral controller with tip mass $=92 \mathrm{~g}(-)$, tip mass $=35 \mathrm{~g}(--)$ and no mass (...).
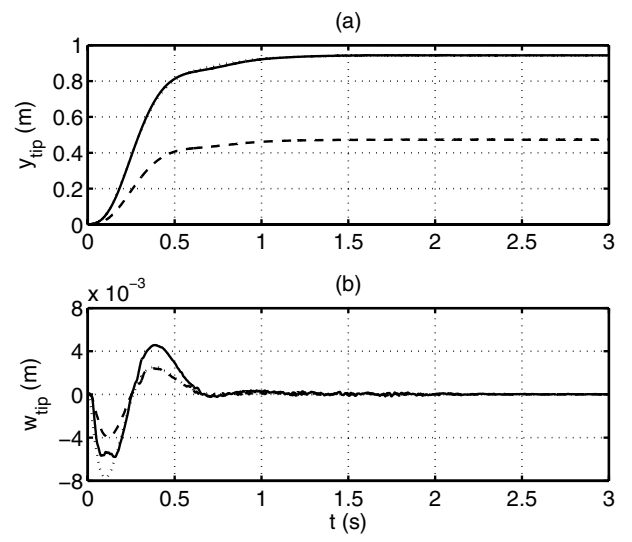

Fig. 14. Time response plots of (a) Tip position $y_{t i p}$, (b) Tip deflection $w_{\text {tip }}$, using augmented Resonant controller $K_{a}(s)$ and integral controller for large step input command, $\frac{\pi L}{2} \mathrm{~m}$. Experimental (-), simulation $(\cdots)$ and small step input command $\frac{\pi L}{4}(--) \mathrm{m}$.

attaching a certain amount of mass to the tip to alter the dynamics and natural frequencies of the flexible manipulator. This test is performed to study closed-loop performance of the controller with a change in payload. Two masses are used here, the first has a weight of $35 \mathrm{~g}$ (which is $14 \%$ of the flexible beam weight) and a second set has a weight of $92 \mathrm{~g}$ (which is $35 \%$ of the flexible beam weight). With these masses at the tip, Fig. 13 shows no elevation in the tip vibrations, but a small overshoot in the $y_{\text {tip }}$ response.

The second robustness test was performed against the size of input commands. Fig. 14 shows no loss of performance in the $y_{\text {tip }}$ and $w_{\text {tip }}$ responses when a larger input command of $\frac{\pi L}{2} \mathrm{~m}$ was used. The $y_{t i p}$ response still has similar rise time, settling time and overshoot regardless of the size of input commands.

\section{CONCLUSIONS}

In this paper frequency-domain system identification was used to model a single link flexible manipulator. The identi- fied models have accurately predicted the frequency and time responses of the flexible manipulator. The transfer-functions characterizing the collocated hub angle $\theta_{h}(t)$ response to the input $u(t)$ and the noncollocated tip position $y_{\text {tip }}(t)$ response to the input $u(t)$ were found to have the same dynamic modes. This allows for the damping of the tip position $y_{t i p}(t)$ response, indirectly, by damping the collocated hub angle $\theta_{h}(t)$ response. A Resonant controller was designed to damp the highly resonant modes of the flexible manipulator. The Resonant controller performed successfully in damping those modes. It was also found that the proposed control scheme was robust to perturbations in the resonance frequencies of the flexible manipulator and the size of input command.

\section{REFERENCES}

[1] A. R. Fraser and R. W. Daniel, Perturbation techniques for flexible manipulators. Kluwer Academic Publisher, 1991.

[2] R. H. Cannon and E. Schmitz, "Initial experiments on the end-point control of a flexible one-link robot," The International Journal of Robotics Research, vol. 3, no. 3, pp. 62-75, Fall 1984.

[3] V. Etxebarria, A. Sanz and I. Lizarraga, "Real-time experimental control of a flexible robotic manipulator using a composite approach," in Proceeding of the 2004 IEEE International Conference on Control Applications, September 2004, pp. 955-960.

[4] D. Farruggio and L. Menini, "Two degrees of freedom $H_{\infty}$ control of a flexible link," in Proceedings of the American Control Conference, June 2000, pp. 2280-2284.

[5] M. Karkoub and K. Tamma, "Modelling and $\mu$-synthesis control of flexible manipulators," Computers and Structures, vol. 79, pp. 543$551,2001$.

[6] R. N. Banavar and P. Dominic, "An LQG/ $H_{\infty}$ controller for a flexible manipulator," IEEE Transactions on Control Systems Technology, vol. 3, no. 4, pp. 409-416, December 1995.

[7] V. A. Spector and H. Flashner, "Modeling and design implications of noncollocated control in flexible systems," Transactions of the ASME Journal of Dynamic Systems, Measurement, and Control, vol. 112, pp. 186-193, June 1990.

[8] Z.-H. Luo, "Direct strain feedback control of flexible robot arms: new theoretical and experimental results," IEEE Transaction on Automatic Control, vol. 38, no. 11, pp. 1610-1622, November 1993.

[9] S. -B. Choi, C. -C. Cheong and H. -C. Shin, "Sliding mode control of vibration in a single-link flexible arm with parameter variations," Journal of Sound and Vibration, vol. 179, no. 5, pp. 737-748, 1995.

[10] H. R. Pota, S. O. R. Moheimani and M. Smith, "Resonant controllers for smart structures," Smart Materials and Structures, vol. 11, pp. 1-8, 2002.

[11] S.O. R. Moheimani and B. J. G. Vautier, "Resonant control of structural vibration using charge-driven piezoelectric actuators," IEEE Transaction on Control System Technology, vol. 13, pp. 1021-1035, 2005

[12] D. Halim and S. O. R. Moheimani, "Spatial resonant control of flexible structures - application to a piezoelectric laminate beam," IEEE Transactions on Control System Technology, vol. 9, pp. 37-53, 2001

[13] M. J. Balas, "Feedback control of flexible structures," IEEE Transactions on Automatic Control, vol. AC-23, no. 4, 1978

[14] N. W. Hagood, W. H. Chung and A. v. Flotow, "Modelling of piezoelectric actuator dynamics for active structural control," Journal of Intelligent Material Systems and Structures, vol. 21, 1990.

[15] K. W. Wang, Structural vibration suppression via parametric control actions - piezoelectric materials with real-time semi-active networks, A. Guran and D. J. Inman, Eds. Singapore: World Scientific, 1995.

[16] S. O. R. Moheimani, A. J. Fleming, and S. Behrens, "On the feedback structure of wideband piezoelectric shunt damping systems," Smart Materials and Structures, vol. 12, pp. 49-56, February 2003.

[17] K. Astrom and T. Hagglund, PID Controllers: Theory, Design, and Tuning. Instrument Society of America, 1995.

[18] R. C. Dorf and R. H. Bishop, Modern Control Systems. Addison Wesley Longman, Inc., 1998. 